Institute and the Rothamsted Experimental Stationand that its close proximity to Harwell continues to be useful in work with radioactive tracers.

One previously neglected area of research is the effect of micro-organisms in the soil on nutrient uptake in root systems. It seems that metabolically active micro-organisms on the surface of roots absorb a great deal of plant nutrient at the expense of the plants, but that nutrient transport in sterile soil is far more efficient, with greater uptake in the centre of the root and more even distribution overall.

At Wantage, radioactive tracers are being used extensively to study nutrient uptake in field conditions, and the comparative efficiency of various types of systems. This supplements work already being carried out on the growth and development of root systems in water culture. One by-product of these studies is a device using the attenuation of beta radiation for measuring the water in soil to a depth of $70 \mathrm{~cm}$. Nylon pads are placed between a radioactive source and a Geiger counter; the attenuation of beta radiation varies with the mass of material between source and counter, so that the water absorbed by the nylon pads can be directly related to the beta radiation recorded at various levels.

\section{Graduates for Industry}

Graduates are 20 per cent more plentiful this year than last, but there has been an increase of only 10 per cent in the number of postgraduate grants awarded by the Science Research Council. Professor Brian Flowers, the chairman of the SRC, has said that compared with 1967 there will be between 600 and 1,000 more good quality graduates in science and technology who will not find grants and will be available for industry.

To what extent has industry benefited from this situation? Most companies have now finished their recruiting campaigns at the universities and have made their offers. But final acceptances have not yet been received and it is too early to judge the exact results for this year. The general impression, however, is that the SRC's policy has had little or no identifiable effect on graduate recruitment.

In the words of one veteran recruiter, "Graduates are hungrier this year and there are a lot of social scientists looking for sales jobs". Most companies have seen more people this year but there has been no marked change in quality. ICI has the impression that higher quality graduates are easier to get, but Shell has noticed no difference in quality from previous years. Twenty per cent more graduates applied for interviews with GEC, but this is in part attributable to the favourable publicity brought about by the recent merger with AET. In recent years GEC has noticed a progressive improvement in the quality of electrical and mechanical engineers from certain universities, but it is hard to detect any alteration in this trend effected by the policy of SRC. Ford finds that graduates who had applied for SRC grants are in general of the same quality as those who had not applied.

A more definite change is apparent in the recruitment of postgraduate students. Several companies have received more applications from postgraduates this year, probably because the slowing down of the expansion of new universities has brought fewer new academic posts on to the market.
The SRC itself does not yet have the figures with which to appraise the results of its policy, but if the impressions so far gained in industry are correct, it seems that some of the graduates disappointed of grants have turned elsewhere than to industry, perhaps to teaching or to various short term jobs.

\section{Technology at Teddington}

THe reorganization of the National Physical Laboratory now seems to be complete, judging by the annual report of the laboratory for 1967 (HMSO, 24s.). The changes have been designed to fit the laboratory more closely to the technological needs of industry, and have involved the division of the NPL into three groups-the measurement group, the materials group and another referred to in the report simply as the "third group" but which can be called, without too much injustice, an engineering sciences group. This third group is concerned with aerodynamies, autonomics, mathematics and ships, and last year took over the hovercraft unit at Hythe. One result of the reorganization has been the setting up of committees to oversee the activities of the NPL-chiefly a steering committee which prepares an annual report and a research programme for the ensuing year, and presents these to a second committee, the visiting board. The function of the visiting board seems to be to convey the report of the steering committee to the Royal Society and to the Ministry of Technology, the twin masters of the NPL. The report now published is the second annual report of the steering committee. The chairman is Professor Brian Flowers, the chairman of the Science Research Council, and he seems quite satisfied with the NPL as it is now, and with the programmes of the groups. The steering committee adds that it has considered the need for a new large high-speed wave-tank for the ship division but realizes that this is not the time to ask for such a costly facility. Professor Flowers, the would-be sponsor of the $300 \mathrm{GeV}$ machine, knows that as well as anybody.

\section{Will Indians Survive?}

THE American Indians are splendid subjects for the study of the adaptation of human communities, as the International Biological Programme (IBP) has quickly recognized. To begin with, the date and place of their arrival is more accurately known than for any other population group. The land bridge across the Bering Strait was open only between 26,000 and 10,000 years ago and the present Indian inhabitants, from the Yukon to Tierra del Fuego, are descended from the migrants who crossed then. The rapid spread across the continent means that a homogeneous ancestor group quickly colonized a great variety of habitats. Although isolation was complete for more than 15,000 years, and although even today there are a few communities in South America still operating a Stone Age economy, the integrity of these groups cannot be expected to last much longer.

This emphasizes the importance of a conference held recently in Washington under the sponsorship of the Pan-American Health Organization and the IBP. With the opportunities for studying the 16 million American Indians in mind, the chairman of the conference, Dr James V. Neel, said that "Ours is the last 